\title{
Organopalladium-Functionalized Dendrimers: Insertion of Palladium(0) into Peripheral Carbon-Iodine Bonds of Carbosilane Dendrimers Derived from Polyols. Crystal Structure of $\mathrm{Si}\left\{\left(\mathrm{CH}_{2}\right)_{3} \mathrm{O}_{2} \mathrm{CC}_{6} \mathrm{H}_{4} \mathrm{I}-4\right\}_{4}$
}

\author{
J ason L. Hoare, ${ }^{\dagger}$ Klaus Lorenz, ${ }^{\ddagger}$ Neldes J . Hovestad, ${ }^{\S}$ Wilberth J . J . Smeets," \\ Anthony L. Spek," Allan J . Canty, ${ }^{\dagger}$ Holger Frey, ${ }^{\ddagger}$ and Gerard van Koten*,\$ \\ Department of Chemistry, University of Tasmania, Hobart, Tasmania, 7001 Australia, \\ Freiburger Materialforschungszentrum FMF, Stefan-Meier-Strasse 31, \\ 79104 Freiburg, Germany, Bijvoet Centre for Biomolecular Research, Laboratory of \\ Crystal and Structural Chemistry, Utrecht University, Padualaan 8, \\ $3584 \mathrm{CH}$ Utrecht, The Netherlands, and Debye Institute, Department of Metal-Mediated \\ Synthesis, Utrecht University, Padualaan 8, $3584 \mathrm{CH}$ Utrecht, The Netherlands
}

Received April 22, $1997^{\otimes}$

\begin{abstract}
A carbosilane dendrimer with 12 peripheral iodoarene groups, $\mathrm{Si}\left\{\left(\mathrm{CH}_{2}\right)_{3}\left\{\mathrm{Si}\left(\mathrm{CH}_{2}\right)_{3} \mathrm{O}_{2} \mathrm{C}\left(\mathrm{C}_{6} \mathrm{H}_{4} \mathrm{l}-\right.\right.\right.$ 4) $\left.\}_{3}\right\}_{4}\left(\mathrm{G}_{1}-\mathrm{Arl}, 5\right)$, and the corresponding $\mathrm{G}_{0}$ model compound, $\mathrm{Si}\left\{\left(\mathrm{CH}_{2}\right)_{3} \mathrm{O}_{2} \mathrm{CC}_{6} \mathrm{H}_{4} \mathrm{I}-4\right\}_{4}\left(\mathrm{G}_{0^{-}}\right.$ $\mathrm{Arl}, 4)$ have been prepared from polyol precursors. These compounds react with $\mathrm{Pd}(\mathrm{dba})_{2} /$ tmeda ( $\mathrm{dba}=$ di benzylideneacetone, tmeda $=\mathrm{N}, \mathrm{N}, \mathrm{N}^{\prime}, \mathrm{N}^{\prime}$-tetramethylethylenediamine) to yiel d periphery-palladated complexes, $\mathrm{Si}\left\{\left(\mathrm{CH}_{2}\right)_{3} \mathrm{O}_{2} \mathrm{C}\left(\mathrm{C}_{6} \mathrm{H}_{4}-4\right) \mathrm{Pdl} \text { (tmeda) }\right\}_{4}\left(\mathrm{G}_{0}-\mathrm{ArPdl}\right.$ (tmeda), 6) and $\mathrm{Si}\left\{\left(\mathrm{CH}_{2}\right)_{3}\left\{\mathrm{Si}\left(\mathrm{CH}_{2}\right)_{3} \mathrm{O}_{2} \mathrm{C}\left(\mathrm{C}_{6} \mathrm{H}_{4}-4\right) \mathrm{Pdl} \text { (tmeda) }\right\}_{3}\right\}_{4}\left(\mathrm{G}_{1}-\mathrm{ArPdl}\right.$ (tmeda), 7). Dendrimer 7 represents the first example of an exclusively $\sigma$-bonded completely periphery-palladated dendrimer. Reactions of the model palladium(II) complexes Pdl $\left(\mathrm{EtO}_{2} \mathrm{C}\left(\mathrm{C}_{6} \mathrm{H}_{4}\right)\right.$-4)(tmeda) (2) and $\mathrm{Pdl}\left(\mathrm{EtO}_{2} \mathrm{C}\left(\mathrm{C}_{6} \mathrm{H}_{4}\right)-4\right)(\mathrm{bpy})(3)$ (bpy $=2,2^{\prime}$-bipyridyl) with the transmetalation reagents $\mathrm{LiMe}$ and $\mathrm{SnMe}_{4}$ were not successful, while no reaction occurred with the related reagent $\mathrm{Sn}(\mathrm{C} \sim \mathrm{N}) \mathrm{Me}_{3}\left[\mathrm{C} \sim \mathrm{N}=8\right.$-(dimethylamino)-1-naphthyl]. The molecular structure of $\mathrm{G}_{0}-\mathrm{Arl}$ (5) has been determined by X-ray crystallography and has $C_{2 v}$ symmetry in the solid state.
\end{abstract}

\section{Introduction}

Dendrimers are currently generating enormous interest in many diverse areas of science and technology, due to the range of applications and interesting materials properties arising from their nanoscale, precise molecular architecture, and the variation in branching and linking moieties incorporated into the structures. ${ }^{1-12}$ Various metals have also been incorporated into dendrimers, as part of the dendritic skeleton and/or as peripheral functionalization. For example, $\mathrm{Cr}, 13 \mathrm{~W}, 7 \mathrm{c}$ $\mathrm{Fe}^{14-16 a, 17} \mathrm{Os} / \mathrm{Ru},{ }^{16-21} \mathrm{Co},{ }^{14 b, 22} \mathrm{Rh}^{23} \mathrm{Ni},{ }^{24} \mathrm{Pd} / \mathrm{Pt}, 8,23,25$ $\mathrm{Cu}^{26} \mathrm{Au},{ }^{7 c, 27} \mathrm{Zn}, 17,28$ and $\mathrm{Ge}^{29}$ have been incorporated,

† University of Tasmania.

₹ Freiburger Materialforschungszentrum.

$\S$ Debye Institute, Utrecht University.

" Bijvoet Centre for Biololecular Research, Utrecht University.

${ }^{\otimes}$ Abstract published in Advance ACS Abstracts, August 15, 1997.

(1) (a) Mekel burger, H.-B.; J aworek, W.; Vögtle, F. Angew. Chem., Int. Ed. Engl. 1992, 31, 1571. (b) I ssberner, J .; Moors, R.; Vögtle, F. Angew. Chem., Int. Ed. Engl. 1994, 33, 2413. (c) Moors, R.; Vögtle, F. Chem. Ber. 1993, 126, 2133. (d) Newkome, G. R.; Moorefield, C. N.; Vögtle, F. Dendritic Molecules: Concepts, Synthesis, Perspectives; VCH: Weinheim, 1996. (e) Advances in Dendritic Macromolecules; Newkome, G. R., Ed. J Al Press: Greenwich, CT, 1994, Vol. 1; 1996 Vol. 3.

(2) (a) Tomalia, D. A.; Naylor, A. M.; Goddard, W. A., III. Angew. Chem., Int. Ed. Engl. 1990, 29, 138. (b) Tomalia, D. A. Sci. Am. 1995 May, 42. (c) Ottaviani, M. F.; Cossu, E.; Turro, N.J.; Tomalia, D. A.J . Am. Chem. Soc. 1995, 117, 4387. (d) Duan, R. G.; Miller, L. L.; Tomalia,

D. A. J . Am. Chem. Soc. 1995, 117, 10783.

(3) Fréchet, J. M. J . Science 1994, 263, 1710

(4) Ardoin, N.; Astruc, D. Bull. Soc. Chim. Fr. 1995, 132, 875.

(5) van der Made, A. W.; van Leeuwen, P. W. N. M. J . Chem. Soc., Chem. Commun. 1992, 1400.

(6) (a) J ansen, J . F. G. A.; de Brabander-van den Berg, E. M. M.; Meijer, E. W. Science 1994, 266, 1226. (b) J ansen, J.F. G. A.; Peerlings, H. W. I.; de Brabander-van den Berg, E. M. M.; Meijer, E. W. Angew. Chem., Int. Ed. Engl. 1995, 34, 1206. with interesting luminescent, ${ }^{18 \mathrm{~d}}$ redox, ${ }^{8,14 \mathrm{~d}}$ and cataIytic ${ }^{8,14 d, 24}$ properties resulting in some cases.

Periphery-functionalized dendrimers have considerable potential in homogeneous catalysis, since their chemical structure provides easily accessible and welldefined reactive sites for their precursors, and their

(7) (a) Launay, N.; Caminade, A.-M.; Lahana, R.; Majoral, J .-P. Angew. Chem., Int. Ed. Engl. 1994, 33, 1589. (b) Launay, N.; Caminade A.-M.; Majoral, J.-P.J . Am. Chem. Soc. 1995, 117, 3282. (c) Slany, M.; Bardaji, M.; Casanove, M.-J .; Caminade, A.-M.; Majoral, J .-P.; Chaudret, B. J . Am. Chem. Soc. 1995, 117, 9764. (d) Launay, N.; Slany, M.; Caminade, A.-M.; Majoral, J .-P. J . Org. Chem. 1996, 61, 3799.

(8) Miedaner, A.; Curtis, C. J .; Barkley, R. M.; DuBois, D. L. Inorg. Chem. 1994, 33, 5482.

(9) Lorenz, K.; Mulhaupt, R.; Frey, H.; Rapp, U.; Mayer-Posner, F. J. Macromol ecules 1995, 28, 6657 .

(10) Sekiguchi, A.; Nanjo, M.; Kabuto, C.; Sakurai, H.J . Am. Chem. Soc. 1995, 117, 4195.

(11) Suzuki, H.; Kimata, Y.; Satoh, S.; Kuriyama, A. Chem. Lett. 1995, 293.

(12) Lambert, J . B.; Pflug, J . L.; Denari, J . M. Organometallics 1996 15,615 .

(13) Lobete, F.; Cuadrado, I.; Casado, C. M.; Alonso, B.; Morán, M.; Losada, J. J . Organomet. Chem. 1996, 509, 109.

(14) (a) Astruc, D. New J. Chem. 1992, 16, 305, (b) Moulines, F. Gloaguen, B.; Astruc, D. Angew. Chem., Int. Ed. Engl. 1992, 31, 458. (c) Moulines, F.; Djakovitch, L.; Boese, R.; Gloaguen, B.; Thiel, W.; Fillaut, J .-L.; Delville, M.-H.; Astruc, D. Angew. Chem., Int. Ed. Engl. 1993, 32, 1075. (d) Fillaut, J .-L.; Linares, J .; Astruc, D. Angew. Chem., Int. Ed. Engl. 1994, 33, 2460.

(15) (a) Alonso, B.; Cuadrado, I.; Moran, M.; Losada, J . J . Chem. Soc., Chem. Commun. 1994, 2575. (b) Alonso, B.; Moran, M.; Casado, C. M.; Lobete, F.; Losada, J .; Cuadrado, I. Chem. Mater. 1995, 7, 1440. (c) Cuadrado, I.; Moran, M.; Casado, C. M.; Alonso, B.; Lobete, F.; Garcia, B.; I bisate, M.; Losada, J . Organometallics 1996, 15, 5278.

(16) (a) Liao, Y. H.; Moss, J . R. J . Chem. Soc., Chem. Commun. 1993 1774. (b) Moss, J . R. Platinum Met. Rev. 1995, 39, 33. (c) Liao, Y.-H.; Moss, J. R. Organometallics 1995, 14, 2130.

(17) Dandliker, P. J .; Diederich, F .; Gisselbrecht, J .-P.; Louati, A.; Gross, M. Angew. Chem., Int. Ed. Engl. 1995, 34, 2725. 
macromolecular size should allow simple removal of catalyst residues from product streams. In contrast to coiled linear macromolecules, dendrimers possess higher overall shape persistence in solution. This allows their easy removal from the reaction solution by ultrafiltration since they cannot reptate through membranes as conventional polymers do. Recently, a carbosilane dendrimer containing 12 peripheral organonickel(II) groups was shown to be catalytically active in the Kharasch addition of polyhalogenoal kanes to carbon-carbon double bonds. ${ }^{24}$ In view of the emerging importance of dendrimers as a new class of materials and the versatility of palladium in both stoichiometric and catalytic organic transformations, we have commenced an investigation into the periphery palladation of carbosilane dendrimers via the insertion of $\mathrm{Pd}(0)$ into the $\mathrm{C}-\mathrm{I}$ bond of iodoarenes. This reaction type is a well-established route to aryl palladium systems. ${ }^{30}$ To date, there have been no reports of dendrimers containing organopalladium moieties bonded directly to the periphery of the dendrimer exclusively via a $\mathrm{Pd}-\mathrm{C} \sigma$-bond.

We describe here the synthesis and characterization of a carbosilane dendrimer with peripheral iodoarene functionality, 5; an X-ray crystallographic study of the $\mathrm{G}_{0}$ model, $\mathrm{Si}\left\{\left(\mathrm{CH}_{2}\right)_{3} \mathrm{O}_{2} \mathrm{CC}_{6} \mathrm{H}_{4} \mathrm{I}-4\right\}_{4}, \mathbf{4}$; and the synthesis of fully periphery-palladated $\mathrm{G}_{0}(\mathbf{6})$ and $\mathrm{G}_{1}$ (7). Mo-

(18) (a) Campagna, S.; Denti, G.; Serroni, S.; Ciano, M.; J uris, A.; Balzani, V. Inorg. Chem. 1992, 31, 2982. (b) Denti, G.; Campagna, S.; Serroni, S.; Ciano, M.; Balzani, V. J . Am. Chem. Soc. 1992, 114, 2944 (c) Serroni, S.; Denti, G.; Campagna, S.; J uris, A.; Ciano, M.; Balzani, V. Angew. Chem., Int. Ed. Engl. 1992, 31, 1493. (d) J uris, A.; Balzani, V.; Campagna, S.; Denti, G.; Serroni, S.; Frei, G.; Güdel, H. U. I norg. Chem. 1994, 33, 1491. (e) Campagna, S.; Denti, G.; Serroni, S.; J uris, A.; Venturi, M.; Ricevuto, V.; Balzani, V. Chem. Eur. J . 1995, 1, 211. (f) Campagna, S.; Giannetto, A.; Serroni, S.; Denti, G.; Trusso, S.; Mallamace, F.; Micali, N. J . Am. Chem. Soc. 1995, 117, 1754.

(19) (a) Newkome, G. R.; Cardullo, F.; Constable, E. C.; Moorefield, C. N.; Thompson, A. M. W. C. J . Chem. Soc., Chem. Commun. 1993, 925. (b) Newkome, G. R.; Güther, R.; Moorefield, C. N.; Cardullo, F.; Echegoyen, L.; Perez-Cordero, E.; Luftmann, H. Angew. Chem., Int. Ed. Engl. 1995, 34, 2023.

(20) (a) Constable, E. C.; Harverson, P. Chem. Commun. 1996, 33. (b) Armspach, D.; Cattalini, M.; Constable, E. C.; Housecroft, C. E.; Phillips, D. Chem. Commun. 1996, 1823. (c) Constable, E. C.; Harverson, P.; Oberholzer, M. Chem. Commun. 1996, 1821.

(21) Turro, C.; Niu, S.; Bossmann, S. H.; Tomalia, D. A.; Turro, N.

J . J . Phys. Chem. 1995, 99, 5512.

(22) Seyferth, D.; Kugita, T.; Rheingold, A. L.; Yap, G. P. A. Organometallics 1995, 14, 5362 .

(23) Bardaji, M.; Kustos, M.; Caminade, A.-M.; Majoral, J .-P.; Chaudret, B. Organometallics 1997, 16, 403.

(24) Knapen, J. W. J .; van der Made, A. W.; de Wilde, J. C.; van Leeuwen, P. W. N. M.; Wijkens, P.; Grove, D. M.; van Koten, G. Nature 1994, 372, 659 .

(25) (a) Achar, S.; Puddephatt, R. J . J . Chem. Soc., Chem. Commun. 1994, 1895. (b) Achar, S.; Puddephatt, R. J . Angew. Chem., Int. Ed. Engl. 1994, 33, 847. (c) Achar, S.; Vittal, J . J .; Puddephatt, R. J . Organometallics 1996, 15, 43. (d) Liu, G.-X.; Puddephatt, R. J . Inorg. Chim. Acta 1996, 251, 319. (e) Ohshiro, N.; Takei, F.; Onitsuka, K.; Takahashi, S. Chem. Lett. 1996, 871. (f) Liu, G.-X.; Puddephatt, R. J. Organometallics 1996, 15, 5257. (g) Huck, W. T. S.; van Veggel, F. C.

J . M.; Reinhoudt, D. N. Angew. Chem., Int. Ed. Engl. 1996, 35, 1213. (h) Huck, W. T. S.; van Veggel, F. C. J . M.; Kropman, B. L.; Blank, D. H. A.; Keim, E. G.; Smithers, M. M. A.; Reinhoudt, D. N. J . Am. Chem. Soc. 1995, 117, 8293.

(26) Ottaviani, M. F.; Bossmann, S.; Turro, N. J .; Tomalia, D. A. J . Am. Chem. Soc. 1994, 116, 661.

(27) (a) Lange, P.; Schier, A.; Schmidbaur, H. Inorg. Chim. Acta 1995, 235, 263. (b) Lange, P.; Schier, A.; Schmidbaur, H. Inorg. Chem. 1996, 35, 637.

(28) Dandliker, P.J .; Diederich, F.; Gross, M.; Knobler, C. B.; Louati, A.; Sanford, E. M. Angew. Chem., Int. Ed. Engl. 1994, 33, 1739.

(29) Huc, V.; Boussaguet, P.; Mazerolles, P. J . Organomet. Chem. 1996, 521, 253.

(30) (a) de Graaf, W.; van Wegen, J .; Boersma, J .; Spek, A. L.; van Koten, G. Recl. Trav. Chim. Pays-Bas 1989, 108, 275. (b) Markies, B. A.; Canty, A. J .; de Graaf, W.; Boersma, J .; J anssen, M. D.; Hogerheide, M. P.; Smeets, W. J . . .; Spek, A. L.; van Koten, G. J . Organomet. Chem. 1994, 482, 191. (c) Kruis, D.; Markies, B. A.; Canty, A. J .; Boersma, J .; van Koten, G. J . Organomet. Chem. 1997, 532, 235.
noorganopalladium(II) complexes containing an ester function have been prepared as models for the palladated dendrimer system for an investigation of transmetalation.

\section{Experimental Section}

Unless stated otherwise, all syntheses were performed under an inert atmosphere using standard Schlenk techniques. Solvents were purified according to standard procedures and freshly distilled before use. The dendrimeric polyol precursors, $\mathrm{Si}\left\{\left(\mathrm{CH}_{2}\right)_{3} \mathrm{OH}\right\}_{4}\left(\mathrm{G}_{0}-\mathrm{OH}\right)$, and $\mathrm{Si}\left\{\left(\mathrm{CH}_{2}\right)_{3}\left\{\mathrm{Si}\left(\mathrm{CH}_{2}\right)_{3} \mathrm{OH}\right\}_{3}\right\}_{4}\left(\mathrm{G}_{1^{-}}\right.$ $\mathrm{OH}),{ }^{9}$ and the transmetal ation reagent $\mathrm{Sn}(\mathrm{C} \sim \mathrm{N}) \mathrm{Me}_{3}[\mathrm{C} \sim \mathrm{N}=$ 8-(dimethylamino)-1-naphthyl] $]^{31}$ were prepared as described. Other reagents were commercial products used as received. NMR spectra were recorded with a Bruker AC300 spectrometer, with chemical shifts given in ppm relative to $\mathrm{SiMe}_{4}$. Microanalyses were performed by Dornis und Kolbe MicroanaIytical Laboratories, Mulheim a.d. Ruhr, Germany, and the Central Science Laboratory, University of Tasmania. A combination of $10^{5}, 10^{4}, 10^{3}$, and $100 \mathrm{~nm}$ columns from Polymer Laboratories was used for GPC studies; chloroform was used as solvent, and data were referenced to narrow polystyrene standards. DSC traces were recorded using a Perkin-EImer 7 Series thermal analysis system.

Synthesis of Model Compounds. Ethyl p-lodobenzoate, $\mathrm{CH}_{3} \mathrm{CH}_{2} \mathrm{O}_{2} \mathrm{CC}_{6} \mathrm{H}_{4} \mathrm{I}-4$ (1). An ethanol solution of $\mathrm{p}$ iodobenzoic acid containing $5 \%$ (wt) concentrated $\mathrm{H}_{2} \mathrm{SO}_{4}$ was refluxed overnight. The solvent was removed by rotary evaporation, and the residue was dissolved in dichloromethane. The dichloromethane solution was washed with aqueous $\mathrm{NaOH}$ and dried over $\mathrm{Na}_{2} \mathrm{SO}_{4}$. Removal of the solvent in vacuo yielded the product as a pale yellow oil. On the basis of its NMR spectra, purification was considered unnecessary. ${ }^{1} \mathrm{H}$ $\operatorname{NMR}\left(\mathrm{CDCl}_{3}\right): \delta 7.69\left(\mathrm{AB}, 3 \mathrm{~J}=8.9 \mathrm{~Hz}, 4, \mathrm{C}_{6} \mathrm{H}_{4}\right), 4.30\left(\mathrm{q},{ }^{3} \mathrm{~J}=\right.$ $\left.7.1 \mathrm{~Hz}, 2, \mathrm{CH}_{2}\right), 1.33\left(\mathrm{t},{ }^{3} \mathrm{~J}=7.1 \mathrm{~Hz}, 3, \mathrm{CH}_{3}\right) .{ }^{13} \mathrm{C}\left\{{ }^{1} \mathrm{H}\right\} \mathrm{NMR}$ $\left(\mathrm{CDCl}_{3}\right): \delta 165.9(\mathrm{OCO}), 137.6\left(\mathrm{C}_{6} \mathrm{H}_{4}\right.$, ortho to $), 131.0\left(\mathrm{C}_{6} \mathrm{H}_{4}\right.$, ortho to OCO), $129.9\left(\mathrm{C}_{6} \mathrm{H}_{4}, \mathrm{CCOOR}\right), 100.7\left(\mathrm{C}_{6} \mathrm{H}_{4}, \mathrm{Cl}\right), 61.2$ $\left(\mathrm{CH}_{2}\right), 14.4\left(\mathrm{CH}_{3}\right)$.

PdI ( $\left.\mathrm{CH}_{3} \mathrm{CH}_{2} \mathrm{O}_{2} \mathrm{CC}_{6} \mathrm{H}_{4}-4\right)$ (tmeda) (2). To a solution of $\mathrm{Pd}(\mathrm{dba})_{2}(0.59 \mathrm{~g}, 1.03 \mathrm{mmol})$ in benzene $(100 \mathrm{~mL})$ were added ethyl p-iodobenzoate $(0.28 \mathrm{~g}, 1.03 \mathrm{mmol})$ in benzene $(5 \mathrm{~mL})$ and tmeda $(0.2 \mathrm{~mL}, 1.3 \mathrm{mmol})$. The solution was stirred at room temperature for $2 \mathrm{~h}$, during which time the col or changed from deep purple to orange. The solution was filtered to remove traces of metallic palladium, the volume was reduced in vacuo, and pentane was added to precipitate the product as a yellow solid, which was washed with pentane/ether (1:1) and collected by centrifugation. Yield: $95 \%$. Mp: $168{ }^{\circ} \mathrm{C} \mathrm{dec}$. Anal. Found: $\mathrm{C}, 36.14 ; \mathrm{H}, 5.10 ; \mathrm{N}, 5.58$. Calcd for $\mathrm{C}_{15} \mathrm{H}_{25} \mathrm{I}-$ $\mathrm{N}_{2} \mathrm{O}_{2} \mathrm{Pd}$ (498.7): C, 36.13; $\mathrm{H}, 5.05 ; \mathrm{N}, 5.62$. ${ }^{1} \mathrm{H} \mathrm{NMR}\left(\mathrm{CDCl}_{3}\right)$ : $\delta 7.58\left(\mathrm{~d},{ }^{3} \mathrm{~J}=8.3 \mathrm{~Hz}, 2, \mathrm{C}_{6} \mathrm{H}_{4}\right.$ ortho to COOR), $7.38\left(\mathrm{~d}, 3^{3} \mathrm{~J}=\right.$ $8.3 \mathrm{~Hz}, 2, \mathrm{C}_{6} \mathrm{H}_{4}$ ortho to Pd), 4.30 (q, 3J = 7.1 Hz, 2, $\mathrm{CH}_{2}$ ), 2.62 and 2.55 (m, 10, $\mathrm{NMe}_{2}$ and $\mathrm{NCH}_{2} \mathrm{CH}_{2} \mathrm{~N}$ overlapping), 2.32 (s, 6, $\left.\mathrm{NMe}_{2}\right), 1.34\left(\mathrm{t}, \mathrm{J}=7.1 \mathrm{~Hz}, 3, \mathrm{CH}_{3}\right) .{ }^{13} \mathrm{C}\left\{{ }^{1} \mathrm{H}\right\} \mathrm{NMR}\left(\mathrm{CDCl}_{3}\right)$ : $\delta 167.8$ (COOR), $156.1(\mathrm{CPd}), 136.5\left(\mathrm{C}_{6} \mathrm{H}_{4}\right), 126.7\left(\mathrm{C}_{6} \mathrm{H}_{4}\right), 125.3$ (CCOOR), $62.2\left(\mathrm{OCH}_{2}\right), 60.3\left(\mathrm{NMe}_{2}\right), 58.4\left(\mathrm{NMe}_{2}\right), 50.0$ and 49.7 $\left(\mathrm{NCH}_{2} \mathrm{CH}_{2} \mathrm{~N}\right), 14.5\left(\mathrm{CH}_{3}\right)$.

PdI ( $\left.\mathrm{CH}_{3} \mathrm{CH}_{2} \mathrm{O}_{2} \mathrm{CC}_{6} \mathrm{H}_{4}-4\right)$ (bpy) (3). A benzene solution of 2 was stirred at room temperature with 1.1 equiv of 2,2'bipyridine (bpy). The solvent was removed in vacuo and the residue was washed with (1:1 vol) pentane/diethyl ether. The yellow solid product was obtained in $100 \%$ yield. Anal. Found: C, 45.18; $\mathrm{H}, 3.68 ; \mathrm{N}, 4.71$. Calcd for $\mathrm{C}_{19} \mathrm{H}_{17} \mathrm{IN}_{2} \mathrm{O}_{2} \mathrm{Pd}$. $0.5 \mathrm{C}_{6} \mathrm{H}_{6}: \mathrm{C}, 45.73 ; \mathrm{H}, 3.50 ; \mathrm{N}, 4.85$. ${ }^{1} \mathrm{H} \mathrm{NMR}\left(\mathrm{CDCl}_{3}\right): \delta 9.63$ $\left(\mathrm{d}, 3 \mathrm{~J}=4.9 \mathrm{~Hz}, 1\right.$, bpy $\left.\mathrm{H}^{6}\right), 8.04(\mathrm{~m}, 4$, bpy), $7.71(\mathrm{~d}, 3 \mathrm{~J}=8.3$ $\mathrm{Hz}, 2, \mathrm{C}_{6} \mathrm{H}_{4}$ ), 7.55 ( $\mathrm{m}, 4, \mathrm{C}_{6} \mathrm{H}_{4}$ and bpy overlapping), 7.35 (" $\mathrm{t}$ ", 1, bpy), $4.35\left(\mathrm{q},{ }^{3} \mathrm{~J}=7.1 \mathrm{~Hz}, 2, \mathrm{CH}_{2}\right), 1.38\left(\mathrm{t}, 3 \mathrm{~J}=7.1 \mathrm{~Hz}, \mathrm{CH}_{3}\right)$. ${ }^{13} \mathrm{C}\left\{{ }^{1} \mathrm{H}\right\} \mathrm{NMR}\left(\mathrm{CDCl}_{3}\right) \delta 153.5(\mathrm{CPd}), 150.5$ (bpy), 139.4, 139.3,

(31) J astrzebski, J . T. B. H.; Boersma, J .; Esch, P.; van Koten, G. Organometallics 1991, 10, 930. 
Table 1. Crystal Data and Details of the Structure Determination of $\mathbf{G}_{0}$-Arl, $\mathrm{Si}\left\{\left(\mathrm{CH}_{2}\right)_{3}\left\{\mathrm{Si}\left(\mathrm{CH}_{2}\right)_{3} \mathrm{O}_{2} \mathrm{C}\left(\mathrm{C}_{6} \mathrm{H}_{4} \mathrm{I}-4\right\}_{3}\right\}_{4}(4)\right.$

\begin{tabular}{|c|c|}
\hline $\begin{array}{l}\text { empirical formula } \\
\text { fw }\end{array}$ & $\begin{array}{l}\mathrm{C}_{40} \mathrm{H}_{40}{ }_{4} \mathrm{O}_{8} \mathrm{Si} \\
1184.46\end{array}$ \\
\hline cryst syst & monodinic \\
\hline space group & C2/c (No. 15) \\
\hline$a, b, c(\AA)$ & $\begin{array}{l}33.165(2), 6.8714(3) \\
23.8827(17)\end{array}$ \\
\hline$\beta$ (deg) & $128.729(7)$ \\
\hline $\begin{array}{l}V_{\text {calcd }}\left(\AA^{3}\right) \\
Z\end{array}$ & $\begin{array}{l}4245.9(6) \\
4\end{array}$ \\
\hline$D_{\text {calcd }}\left(\mathrm{g} / \mathrm{cm}^{3}\right)$ & $\begin{array}{l}4 \\
1.853\end{array}$ \\
\hline $\mathrm{F}(000)$ (electrons) & 2280 \\
\hline$\mu(\mathrm{M} \circ \mathrm{K} \alpha)\left(\mathrm{cm}^{-1}\right)$ & 30.1 \\
\hline cryst size (mm) & $0.05 \times 0.20 \times 0.30$ \\
\hline temp $(\mathrm{K})$ & 150 \\
\hline $\begin{array}{l}\text { radiation, Mo K } \alpha \text { (graphite } \\
\text { monochromated) }(\AA)\end{array}$ & 0.71073 \\
\hline$\theta_{\min }, \theta_{\max }(\mathrm{deg})$ & $1.6,27.5$ \\
\hline scan type & $\omega$ \\
\hline scan (deg) & $0.64+0.35 \tan \theta$ \\
\hline no. of ref refln(s) & 3 , decay $1.8 \%$ \\
\hline data set & -42: 38; 0: 8;-27: 30 \\
\hline $\begin{array}{l}\text { total no. of unique data } \\
\text { no. of obsd data }(1>2.0 \sigma(1))\end{array}$ & $\begin{array}{l}8371,4839 \\
3616\end{array}$ \\
\hline $\mathrm{N}_{\text {ref }}, \mathrm{N}_{\text {par }}$ & 4839,254 \\
\hline$R, w R, S$ & $0.0337,0.0758,1.01$ \\
\hline weighting scheme & $\begin{array}{l}\mathrm{w}^{-1}=\sigma^{2}\left(\mathrm{~F}_{\mathrm{o}}^{2}\right)+0.0325 \mathrm{P}^{2}+ \\
6.2190 \mathrm{P}\left[\mathrm{P}=\left(\mathrm{F}_{\mathrm{o}}^{2}+2 \mathrm{~F}_{\mathrm{c}}^{2}\right) / 3\right]\end{array}$ \\
\hline max and av shift/error & $0.00,0.00$ \\
\hline $\begin{array}{l}\text { min and max resd } \\
\text { intensity }\left(e / \AA^{3}\right)\end{array}$ & $-0.90,0.70$ \\
\hline
\end{tabular}

137.2, 137.0, 128.8, 128.0, 127.4, 127.2, 127.1, 126.3, 122.7, 122.2, $60.9\left(\mathrm{CH}_{2}\right), 15.0\left(\mathrm{CH}_{3}\right)$.

Synthesis of $\mathbf{G}_{0}$-Arl and $\mathbf{G}_{1}-\mathrm{Arl}$. $\mathbf{G}_{0}-\mathrm{Arl}, \mathrm{Si}\left\{\left(\mathrm{CH}_{2}\right)_{3} \mathrm{O}_{2^{-}}\right.$ $\left.\mathbf{C C}_{6} \mathbf{H}_{4} \mathbf{I}-4\right\}_{4}$ (4). p-l odobenzoic acid (1.576 g, $\left.6.354 \mathrm{mmol}\right)$ and carbonyldiimidazole (CDI) $(0.957 \mathrm{~g}, 5.902 \mathrm{mmol})$ were dissolved in dry $\mathrm{N}, \mathrm{N}$-dimethylformamide (DMF) $(10 \mathrm{~mL})$. The solution was stirred for $1.5 \mathrm{~h}$ at ambient temperature and subsequently heated at $60{ }^{\circ} \mathrm{C}$ for $12 \mathrm{~h}$. $\mathrm{Si}\left(\mathrm{CH}_{2} \mathrm{CH}_{2} \mathrm{CH}_{2} \mathrm{OH}\right)_{4}$ $\left(\mathrm{G}_{0}-\mathrm{OH}\right)(0.3 \mathrm{~g}, 1.135 \mathrm{mmol})$ was dissolved in dry DMF $(5 \mathrm{~mL})$ and added to the solution of the acid imidazolide. The reaction mixture was kept at $80{ }^{\circ} \mathrm{C}$ for $44 \mathrm{~h}$. The DMF was removed in vacuo, and the remaining solid was redissolved in $\mathrm{CH}_{2} \mathrm{Cl}_{2}$ and filtered. The filtrate was washed twice with saturated aqueous $\mathrm{NaHCO}_{3}$ and twice with water. After drying over $\mathrm{Na}_{2} \mathrm{SO}_{4}$ and evaporation of the solvent the crude product was recrystallized from acetone/ $\mathrm{CHCl}_{3}(8: 1)$ to yield the product as a crystalline white solid $(0.82 \mathrm{~g}, 61 \%)$. Crystals suitable for X-ray analysis were obtained from acetone/ $\mathrm{CHCl}_{3}(5: 1)$ at $4{ }^{\circ} \mathrm{C}$. Anal. Found: $\mathrm{C}, 40.55 ; \mathrm{H}, 3.36 ; \mathrm{I}, 42.65$. Calcd for $\mathrm{C}_{40} \mathrm{H}_{40} \mathrm{l}_{4} \mathrm{O}_{8} \mathrm{Si}$ (1184.5): C, 40.56; $\mathrm{H}, 3.40 ; \mathrm{I}, 42.86$. ${ }^{1} \mathrm{H} N M R$ $\left(\mathrm{CDCl}_{3}\right): \delta 0.70\left(\mathrm{~m}, 8, \mathrm{SiCH}_{2}\right), 1.78\left(\mathrm{~m}, 8, \mathrm{SiCH}_{2} \mathrm{CH}_{2}\right), 4.26(\mathrm{t}$, 3) $\left.=6.8 \mathrm{~Hz}, 8, \mathrm{CH}_{2} \mathrm{O}\right), 7.71\left(\mathrm{~d},{ }^{3} \mathrm{~J}=6.8 \mathrm{~Hz}, 8, \mathrm{C}_{6} \mathrm{H}_{4}\right), 7.77(\mathrm{~d}$, 3j $\left.=6.8 \mathrm{~Hz}, 8, \mathrm{C}_{6} \mathrm{H}_{4}\right) .{ }^{13} \mathrm{C}\left\{{ }^{1} \mathrm{H}\right\} \mathrm{NMR}\left(\mathrm{CDCl}_{3}\right): \delta 8.0(\mathrm{SiC}), 23.2$ $\left(\mathrm{SiCH}_{2} \mathrm{CH}_{2}\right), 67.5\left(\mathrm{SiCH}_{2} \mathrm{CH}_{2} \mathrm{CH}_{2}\right), 100.8(\mathrm{Cl}), 129.8(\mathrm{OC}(\mathrm{O}) \mathrm{C})$, 131.0 (Ar, C meta to $\mathrm{Cl}$ ), 137.8 (Ar, C ortho to $\mathrm{Cl}$ ), 166.0 (OC(O)). Melting point: $129^{\circ} \mathrm{C}$.

$\mathbf{G}_{1}-\mathrm{Arl}, \mathrm{Si}\left\{\left(\mathrm{CH}_{2}\right)_{3}\left\{\mathrm{Si}\left(\mathrm{CH}_{2}\right)_{3} \mathrm{O}_{2} \mathbf{C}\left(\mathrm{C}_{6} \mathrm{H}_{4} \mathrm{I}-4\right)\right\}_{3}\right\}_{4}$ (5). To a solution of $\mathrm{G}_{1}-\mathrm{OH}(0.350 \mathrm{~g}, 0.344 \mathrm{mmol})$ in pyridine $(8 \mathrm{~mL})$ cooled in an ice/water bath was added dropwise a solution of 4-iodobenzoyl chloride $(1.414 \mathrm{~g}, 5.306 \mathrm{mmol})$ in THF (12 mL). The reaction mixture was allowed to warm to ambient temperature, was stirred for 3 days, and then was poured into water $(150 \mathrm{~mL})$ containing concentrated $\mathrm{HCl}(9 \mathrm{~mL})$. The resulting suspension was extracted with $\mathrm{CH}_{2} \mathrm{Cl}_{2}(300 \mathrm{~mL})$, and the organic layer was washed with a saturated solution of $\mathrm{NaHCO}_{3}$ and finally water. After drying over $\mathrm{Na}_{2} \mathrm{SO}_{4}$ and filtration, the solvent was removed and the residue was extracted several times with acetone. The remaining solid was dissolved in $\mathrm{CHCl}_{3}$, and insoluble material was removed by filtration. The solvent was removed, and the residue was purified by chromatography on silica using $\mathrm{CH}_{2} \mathrm{Cl}_{2} /$ diethyl

\section{Scheme 1a}
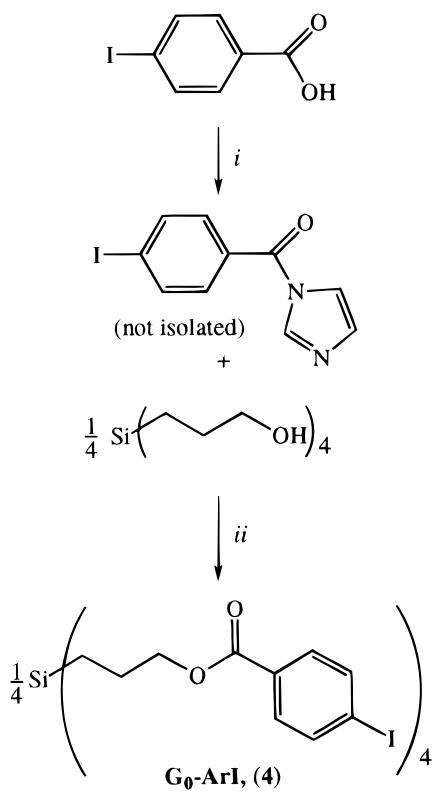

a (i) carbonyl diimi dazole, -imidazole, $-\mathrm{CO}_{2}, \mathrm{DMF}, 60^{\circ} \mathrm{C}$, $12 \mathrm{~h}$; (ii) -imidazole, DMF, $80^{\circ} \mathrm{C}, 44 \mathrm{~h}$.

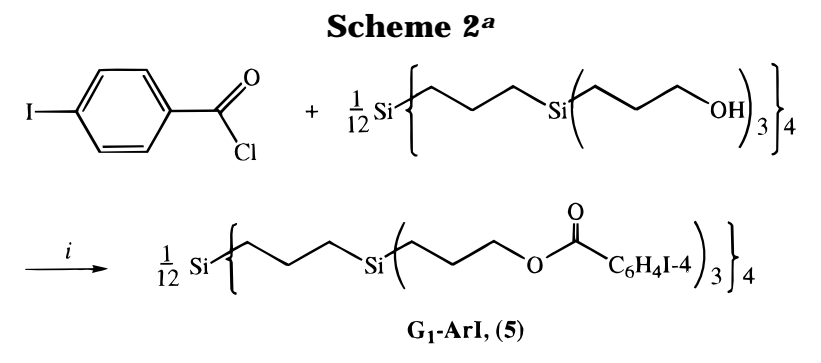

a (i) $-\mathrm{HCl}$, pyridine $/ \mathrm{THF}, 0^{\circ} \mathrm{C}$ to room temperature, 3 days.

ether (100:1 vol) as eluent, yiel ding glassy $\mathrm{G}_{1}-\mathrm{Arl}(0.52 \mathrm{~g}, 40 \%)$. Anal. Found: $\mathrm{C}, 41.97 ; \mathrm{H}, 4.13$. Calcd for $\mathrm{C}_{132} \mathrm{H}_{144} \mathrm{l}_{12} \mathrm{O}_{24} \mathrm{Si}_{5}$ (3777.8): C, 41.97; $\mathrm{H}, 3.84 .{ }^{1} \mathrm{H} N M R\left(\mathrm{CDCl}_{3}\right): \delta 0.60(\mathrm{~m}, 40$, $\left.\mathrm{SiCH}_{2}\right), 1.31\left(\mathrm{~m}, 8, \mathrm{SiCH}_{2} \mathrm{CH}_{2} \mathrm{CH}_{2} \mathrm{Si}\right), 1.73\left(\mathrm{~m}, 24, \mathrm{SiCH}_{2-}\right.$ $\mathrm{CH}_{2} \mathrm{CH}_{2} \mathrm{O}$ ), 4.22 (t, 3) $=6.8 \mathrm{~Hz}, 24, \mathrm{RCH}_{2} \mathrm{O}$ ), 7.72 (d, 3) $=6.4$ $\mathrm{Hz}, 24, \mathrm{C}_{6} \mathrm{H}_{4}$, ortho to COOR), $7.65\left(\mathrm{~d}, \mathrm{~J}=6.4 \mathrm{~Hz}, 24, \mathrm{C}_{6} \mathrm{H}_{4}\right.$ ortho to I). ${ }^{13} \mathrm{C}\left\{{ }^{1} \mathrm{H}\right\}$ NMR $\left(\mathrm{CDCl}_{3}\right): \delta 165.8$ (COOR), 137.7 $\left(\mathrm{C}_{6} \mathrm{H}_{4}\right.$, ortho to I ), $130.8\left(\mathrm{C}_{6} \mathrm{H}_{4}\right.$, meta to I $), 129.6\left(\mathrm{C}_{6} \mathrm{H}_{4}\right.$, para to I), $100.8(\mathrm{Cl}), 67.5\left(\mathrm{CH}_{2} \mathrm{O}\right), 23.2\left(\mathrm{SiCH}_{2} \mathrm{CH}_{2} \mathrm{CH}_{2} \mathrm{O}\right), 18.5-$ 17.2 (carbons of the inner core), $7.9\left(\mathrm{SiCH}_{2} \mathrm{CH}_{2} \mathrm{CH}_{2} \mathrm{O}\right)$.

Synthesis of Palladated $\mathbf{G}_{0}$ and $\mathbf{G}_{1}$. $\mathbf{G}_{0}$-ArPdI (tmeda), $\mathrm{Si}\left\{\left(\mathrm{CH}_{2}\right)_{3} \mathrm{O}_{2} \mathbf{C}\left(\mathrm{C}_{6} \mathrm{H}_{4}-\mathbf{4}\right) \mathrm{PdI} \text { (tmeda) }\right\}_{4}$ (6). To a solution of $\mathrm{Pd}(\mathrm{dba})_{2}(0.19 \mathrm{~g}, 0.33 \mathrm{mmol})$ in THF $(50 \mathrm{~mL})$ were added $\mathrm{G}_{0^{-}}$ Arl (4; $95.5 \mathrm{mg}, 0.081 \mathrm{mmol})$ in THF (5 mL) and tmeda $(0.1 \mathrm{~mL}, 0.66 \mathrm{mmol})$. The solution was stirred at room temperature for $1 \mathrm{~h}$ and filtered, and the sol vent was removed in vacuo. The residue was washed with pentane/diethyl ether (1:1) and dried in vacuo to give the yellow solid product in $95 \%$ yield. Anal. Found: $\mathrm{C}, 37.13 ; \mathrm{H}, 4.72 ; \mathrm{N}, 5.00$. Calcd for $\mathrm{C}_{64} \mathrm{H}_{104} \mathrm{l}_{4} \mathrm{~N}_{8} \mathrm{O}_{8} \mathrm{Pd}_{4} \mathrm{Si}$ (2075.0): C, 37.05; $\mathrm{H}, 5.05 ; \mathrm{N}, 5.40$. ${ }^{1} \mathrm{H}$ NMR $\left(\mathrm{CDCl}_{3}\right): \delta 7.57\left(\mathrm{~d},{ }^{3} \mathrm{~J}=8.3 \mathrm{~Hz}, 8, \mathrm{C}_{6} \mathrm{H}_{4} \mathrm{H}_{2,6}\right), 7.42\left(\mathrm{~d},{ }^{3} \mathrm{~J}\right.$ $\left.=8.2 \mathrm{~Hz}, 8, \mathrm{C}_{6} \mathrm{H}_{4} \mathrm{H}_{3,5}\right), 4.20\left(\mathrm{t}, 3 \mathrm{~J}=6.5 \mathrm{~Hz}, 8, \mathrm{CH}_{2} \mathrm{O}\right), 2.71-$ 2.55 and 2.68 (m, 32, $\mathrm{NMe}_{2}$ and $\mathrm{NCH}_{2}$ overlapping), 2.55 (m, 8, $\left.\mathrm{NCH}_{2}\right), 2.26\left(\mathrm{~s}, 24, \mathrm{NMe}_{2}\right), 1.74\left(\mathrm{~m}, 8, \mathrm{SiCH}_{2} \mathrm{CH}_{2}\right), 0.71(\mathrm{~m}$ 8, $\left.\mathrm{SiCH}_{2}\right) .{ }^{13} \mathrm{C}\left\{{ }^{1} \mathrm{H}\right\} N M R\left(\mathrm{CDCl}_{3}\right): \delta 167.7$ (COOR), 156.6 (CPd), 136.6 (Ar), 126.6 (Ar), 125.1 (CCOOR), $66.8\left(\mathrm{CH}_{2} \mathrm{O}\right)$, $62.2\left(\mathrm{NMe}_{2}\right), 58.4\left(\mathrm{NMe}_{2}\right), 50.0$ and $49.9\left(\mathrm{NCH}_{2} \mathrm{CH}_{2} \mathrm{~N}\right), 23.3$ $\left(\mathrm{SiCH}_{2} \mathrm{CH}_{2}\right), 8.1\left(\mathrm{SiCH}_{2}\right)$.

$\mathrm{G}_{1}$-ArPdl (tmeda), $\mathrm{Si}\left\{\left(\mathrm{CH}_{2}\right)_{3}\left\{\mathrm{Si}\left(\mathrm{CH}_{2}\right)_{3} \mathrm{O}_{2} \mathrm{C}\left(\mathrm{C}_{6} \mathrm{H}_{4}-\mathbf{4}\right) \mathrm{PdI}-\right.\right.$ (tmeda) $\left.\}_{3}\right\}_{4}$ (7). To a solution of $\mathrm{G}_{1}-\mathrm{Arl}(0.162 \mathrm{~g}, 0.043 \mathrm{mmol})$ in THF (25 mL) were added tmeda ( $15 \mu \mathrm{L}, 0.099 \mathrm{mmol})$ and $\mathrm{Pd}(\mathrm{dba})_{2}(0.050 \mathrm{~g}, 0.087 \mathrm{mmol})$. The resulting purple solution was stirred at room temperature for $30 \mathrm{~min}$, during which time 


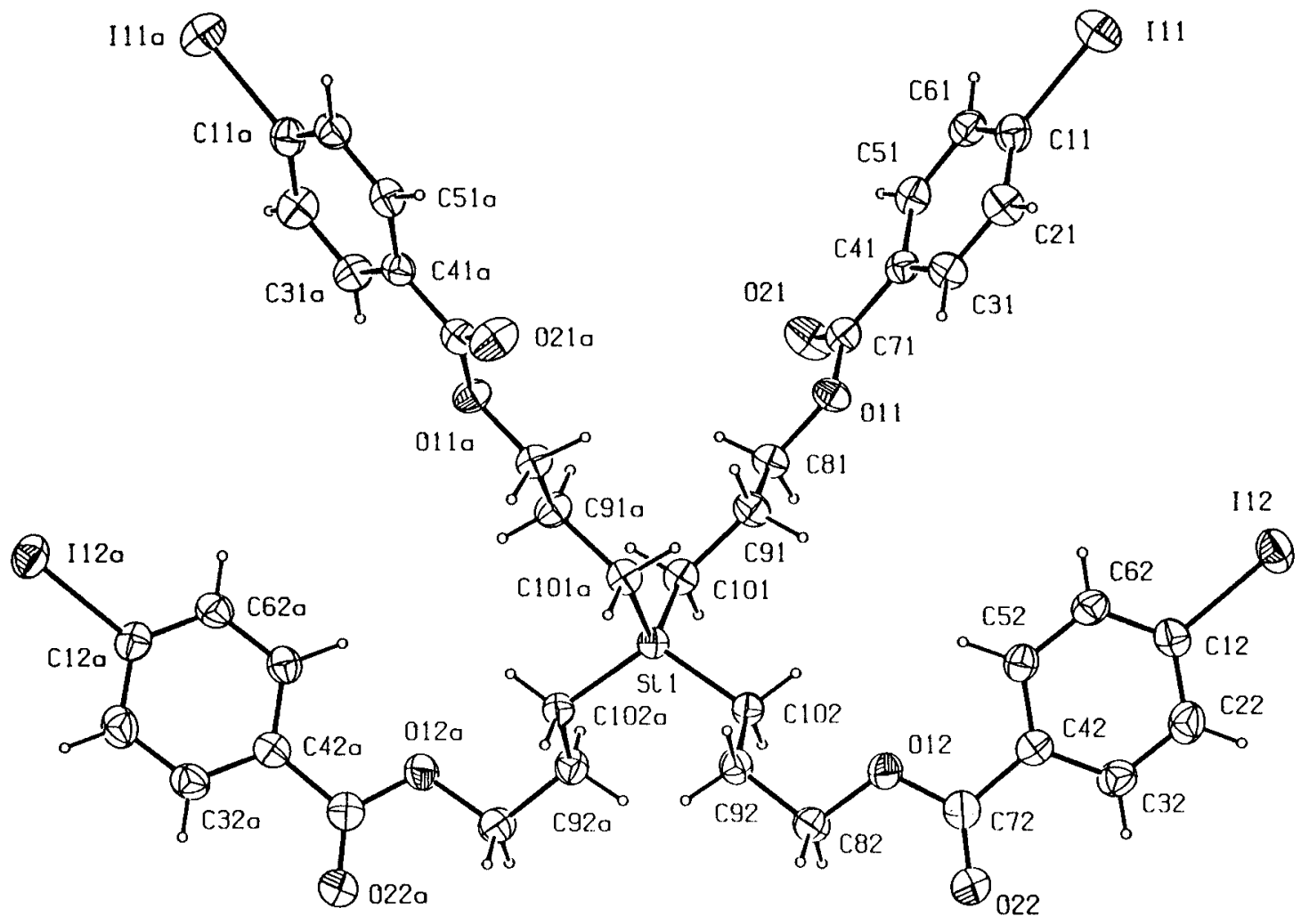

Figure 1. ORTEP drawing (50\% probability level) of $\mathrm{Si}\left\{\left(\mathrm{CH}_{2}\right)_{3} \mathrm{O}_{2} \mathrm{CC}_{6} \mathrm{H}_{4} \mathrm{I}-4\right\}_{4}$ (4).

the color changed to bright orange. The THF was removed in vacuo, and the orange residue was extracted with $\mathrm{CH}_{2} \mathrm{Cl}_{2}$ and filtered. The solvent was removed and the residue washed with diethyl ether $(4 \times 5 \mathrm{~mL})$ and then stirred in diethyl ether for 3 days, during which time the oily residue became a powdery solid, which was collected by centrifugation and dried in vacuo $(0.22 \mathrm{~g}, 81 \%)$. Anal. Found: $\mathrm{C}, 38.09 ; \mathrm{H}, 5.25 ; \mathrm{N}$, 5.23. Calcd for $\mathrm{C}_{204} \mathrm{H}_{336} \mathrm{I}_{12} \mathrm{~N}_{24} \mathrm{O}_{24} \mathrm{Si}_{5} \mathrm{Pd}_{12}$ (6449): C, 37.99; $\mathrm{H}$, 5.25; $\mathrm{N}, 5.21$. ${ }^{1} \mathrm{H}$ NMR $\left(\mathrm{CD}_{2} \mathrm{Cl}_{2}\right): \delta 7.52\left(\mathrm{~d},{ }^{3} \mathrm{~J}=8.2 \mathrm{~Hz}, 24\right.$, $\mathrm{C}_{6} \mathrm{H}_{4} \mathrm{H}_{2,6}$ ), $7.37\left(\mathrm{~d}, 3 \mathrm{~J}=8.2 \mathrm{~Hz}, 24, \mathrm{C}_{6} \mathrm{H}_{4} \mathrm{H}_{3,5}\right), 4.15$ (t, 3 $\mathrm{J}=$ $6.0 \mathrm{~Hz}, 24, \mathrm{CH}_{2} \mathrm{O}$ ), 2.63 (s, 72, $\left.\mathrm{NMe}_{2}\right), 2.55$ (s, $\left.48 \mathrm{NCH}_{2}\right), 2.21$ (s, 72, $\left.\mathrm{NMe}_{2}\right), 1.69\left(\mathrm{~m}, 24, \mathrm{SiCH}_{2} \mathrm{CH}_{2}\right), 1.34\left(\mathrm{~m}, 8, \mathrm{SiCH}_{2} \mathrm{CH}_{2^{-}}\right.$ $\left.\mathrm{CH}_{2} \mathrm{Si}\right), 0.65\left(\mathrm{~m}, 40, \mathrm{SiCH}_{2}\right) .{ }^{13} \mathrm{C}\left\{{ }^{1} \mathrm{H}\right\} \mathrm{NMR}\left(\mathrm{CD}_{2} \mathrm{Cl}_{2}\right): \delta 167.6$ (COOR), 157.6 (CPd), 137.0 (Ar), 126.6 (Ar), 125.4 (CCOOR), $67.3\left(\mathrm{CH}_{2} \mathrm{O}\right), 62.4\left(\mathrm{NMe}_{2}\right), 58.6\left(\mathrm{NMe}_{2}\right), 50.2$ and $50.0\left(\mathrm{NCH}_{2-}\right.$ $\left.\mathrm{CH}_{2} \mathrm{~N}\right), 23.7\left(\mathrm{SiCH}_{2} \mathrm{CH}_{2}\right), 8.4\left(\mathrm{SiCH}_{2}\right), 18.8-15.4$ (carbons of the inner core).

Attempts To Transmetalate Pd(II) Model Complexes 2 and 3. With MeLi. Pdl $\left(\mathrm{EtO}_{2} \mathrm{CC}_{6} \mathrm{H}_{4}-4\right.$ )(tmeda) was stirred in ether at $-40{ }^{\circ} \mathrm{C}$ with 1 equiv of MeLi. Decomposition occurred before and during workup at $0{ }^{\circ} \mathrm{C}$.

With SnMe 4 . Pdl ( $\mathrm{EtO}_{2} \mathrm{CC}_{6} \mathrm{H}_{4} 4$ )(tmeda) was stirred in THF at room temperature with 1 equiv of $\mathrm{SnMe}_{4}$ for 2 days. After removal of solvent in vacuo and washing of the residue with diethyl ether, $\mathrm{NMR}$ analysis $\left(\mathrm{CDCl}_{3}\right)$ showed no conversion to a di organopalladium(II) species. $\mathrm{Pdl}\left(\mathrm{EtO}_{2} \mathrm{CC}_{6} \mathrm{H}_{4}-4\right)$ (bpy) was stirred in $\mathrm{THF}$ with 1 equiv of $\mathrm{SnMe}_{4}$ overnight at room temperature and then at reflux for $2 \mathrm{~h}$. After removal of the solvent and washing of the residue with diethyl ether, NMR analysis $\left(\mathrm{CDCl}_{3}\right)$ showed no conversion to a diorganopalladium(II) species.

With (8-(Dimethylamino)-1-naphthyl)trimethyltin, Sn(C $\sim \mathbf{N}) \mathbf{M e}_{3} . \quad \mathrm{Pdl}\left(\mathrm{EtO}_{2} \mathrm{CC}_{6} \mathrm{H}_{4}-4\right)$ (tmeda) was stirred overnight in THF at room temperature with 1 equiv of $\mathrm{Sn}(\mathrm{C} \sim \mathrm{N}) \mathrm{Me}_{3}$. After removal of the solvent, NMR analysis (toluene- $\mathrm{d}_{8}$ ) showed no conversion to $\mathrm{Sn}(\mathrm{C} \sim \mathrm{N}) \mathrm{Me}_{2} \mathrm{l}$. $\mathrm{Pdl}\left(\mathrm{EtO}_{2} \mathrm{CC}_{6} \mathrm{H}_{4^{-}}\right.$ 4)(bpy) was stirred with 1 equiv of $\mathrm{Sn}(\mathrm{C} \sim \mathrm{N}) \mathrm{Me}_{3}$ in $\mathrm{THF}$ at room temperature for 3 days. After removal of the solvent, NMR analysis (toluene- $d_{8}$ ) showed no conversion to $S n(C \sim N)$ $M e_{2} l$. The residue was washed with toluene, and NMR

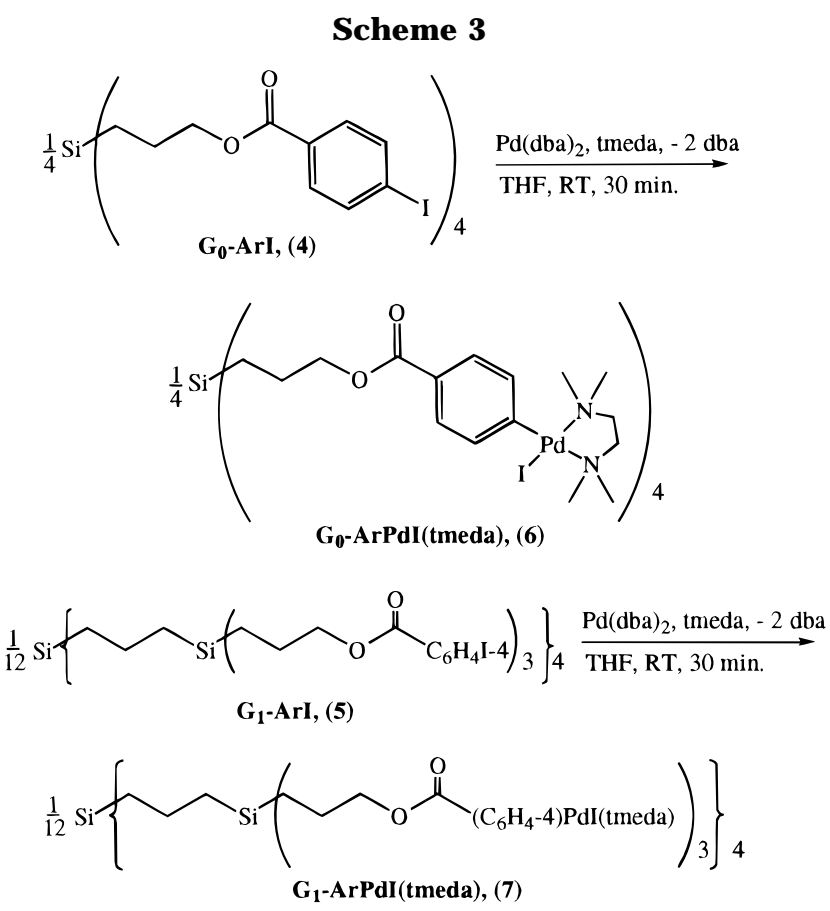

analysis $\left(\mathrm{CDCl}_{3}\right)$ showed no conversion to a diorganopalladium(II) species.

$\mathrm{Pdl}\left(\mathrm{EtO}_{2} \mathrm{CC}_{6} \mathrm{H}_{4}-4\right)$ (bpy) was stirred in THF with $\mathrm{Sn}(\mathrm{C} \sim \mathrm{N}) \mathrm{Me}_{3}$ in THF at reflux for $2 \mathrm{~h}$, during which time decomposition to metallic palladium occurred. After workup, NMR analysis (toluene- $\mathrm{d}_{8}$ ) showed no conversion to $\mathrm{Sn}(\mathrm{C} \sim \mathrm{N}) \mathrm{Me} \mathrm{e}_{2}$.

X-ray Structure Determination. A transparent colorless plate-shaped crystal was glued to the tip of a Lindemann glass capillary (inert oil technique) and transferred into the cold nitrogen stream of an Enraf-N onius CAD4T diffractometer on a rotating anode. Accurate unit-cell parameters were derived from the setting angles of 25 reflections (SET4 method). ${ }^{32 a}$ Crystal data and details of the structure determination are presented in Table 1. An empirical correction for absorption 


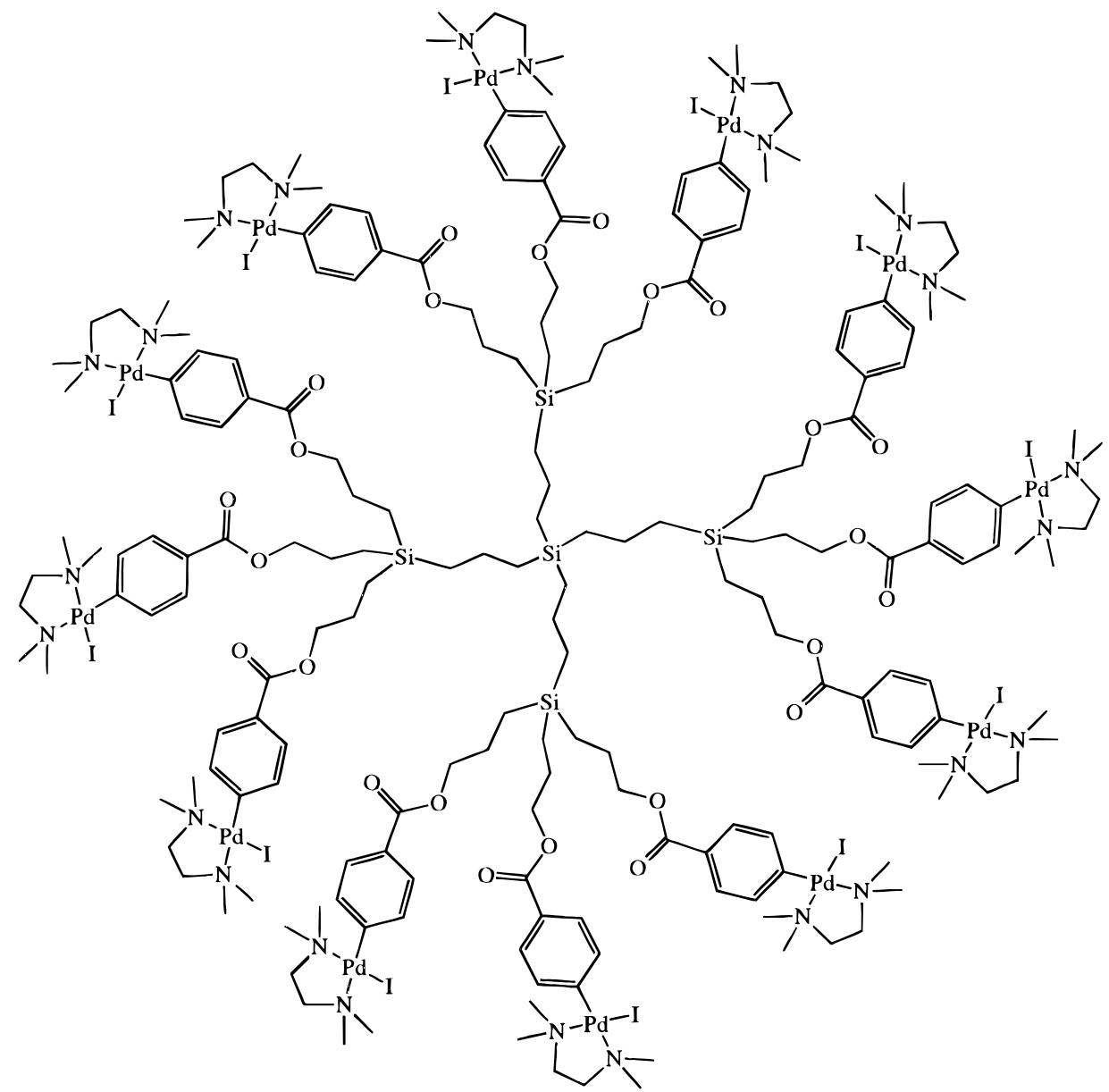

Figure 2. $\mathrm{G}_{1}-\mathrm{ArPdl}$ (tmeda) (7).

was done with the DIFABS technique ${ }^{32 b}$ as implemented in PLATON ${ }^{32 c}$ (correction range $0.614-1.374$ ). The structure was solved with automated Patterson techniques using DIRDIF ${ }^{32 d}$ and refined on $\mathrm{F}^{2}$ by full matrix least-squares (SHELXL-96). ${ }^{32 \mathrm{e}}$ Hydrogen atoms were taken into account at calculated positions riding on their carrier atoms. The highest residual features in the final difference map were near Pd. Neutral atom scattering factors were taken from the International Tables for Crystallography. ${ }^{32 f}$ Geometrical calculations and the ORTEP illustration were done with PLATON. ${ }^{32 c}$

\section{Results and Discussion}

Synthesis and Characterization of $\mathbf{G}_{0}-\mathbf{A r l}$ and $\mathbf{G}_{1}$-Arl. Initial attempts to generate $\mathrm{G}_{0}$ and $\mathrm{G}_{1}$ with peripheral iodoarene groups focused on the generation of the imidazol ide-activated derivative of 4-iodobenzoic acid, followed by esterification with the polyol starting material, and the synthesis of $\mathrm{G}_{0}$-Arl was successfully achieved in good yield using this method (Scheme 1).

Other products of this reaction $\left(\mathrm{CO}_{2}\right.$, imidazole) and excess carboxylic acid were easily removed by washing with acetone, in which the dendrimeric ester is insoluble. This method was also used to synthesize $\mathrm{G}_{1^{-}}$

(32) (a) de Boer, J . L.; Duisenberg, A. J . M. Acta Crystallogr. A 1984, 40, C-410. (b) Walker, N.; Stuart, D. Acta Crystallogr. A 1983, 39, 158. (c) Spek, A. L. Acta Crystallogr. A 1983, 46, C-34. (d) Beurskens, P. T.; Admiraal, G.; Beurskens, G.; Bosman, W. P.; Garcia-Grande, S.; Gould, R. O.; Smits, J. M. M.; Smykalla, C. The DIRDIF program system: Technical report of the Crystallographic laboratory; University of Nijmegen: The Netherlands, 1996. (e) Sheldrick, G. M. SHELXL96. Program for crystal structure refinement; University of Göttingen: Göttingen, Germany, 1996. (f) Wilson, A. J . C., Ed. International Tables for Crystallography; Kluwer Academic Publishers: Dordrecht, The Netherlands, 1992; Vol. C.
Table 2. Selected Bond Distances $(\AA)$ and Angles (deg) and Other Data for $\mathrm{G}_{0}$-Arl, $\left.\mathrm{Si}\left\{\left(\mathrm{CH}_{2}\right)_{3} \mathrm{O}_{2} \mathrm{CC}_{6} \mathrm{H}_{4} \mathrm{I}-4\right\}_{3}\right\}_{4}$ (4)

Bond Distances

\begin{tabular}{llll}
\hline $\mathrm{n}=1$ & $\mathrm{n}=2$ & $\mathrm{n}=1$ & $\mathrm{n}=2$ \\
\hline
\end{tabular}

Si(1)-C(10n) 1.868(4) 1.869(5) C(4n)-C(7n) $1.475(6) 1.495(7)$ C(1n)-I(1n) 2.095(5) 2.097(5) C(5n)-C(6n) 1.382(6) $1.387(7)$

$\mathrm{C}(1 \mathrm{n})-\mathrm{C}(2 \mathrm{n}) \quad 1.388(6) \quad 1.378(8) \mathrm{C}(7 n)-\mathrm{O}(1 \mathrm{n}) \quad 1.336(5) 1.332(6)$

$C(1 n)-C(6 n) \quad 1.379(6) 1.380(6) C(7 n)-O(2 n) \quad 1.210(5) 1.207(5)$

$\mathrm{C}(2 \mathrm{n})-\mathrm{C}(3 \mathrm{n}) \quad 1.380(7) 1.393(7) \mathrm{C}(8 \mathrm{n})-\mathrm{C}(9 \mathrm{n}) \quad 1.505(5) 1.506(7)$

$C(3 n)-C(4 n) \quad 1.391(5) 1.377(6) C(8 n)-O(1 n) \quad 1.448(5) 1.448(6)$

C(4n)-C(5n) 1.389(5) 1.387(7) C(9n)-C(10n) 1.527(7) 1.531(5)

Bond Angles at Silicon

$\mathrm{C}(101)-\mathrm{Si}(1)-\mathrm{C}(102) \quad 109.0(2) \mathrm{C}(101 \mathrm{a})-\mathrm{Si}(1)-\mathrm{C}(102) \quad 107.6(2)$ C(101)-Si(1)-C(101a) 112.7(2) C(102)-Si(1)-C(102a) 111.1(2) $\mathrm{C}(101)-\mathrm{Si}(1)-\mathrm{C}(102 \mathrm{a})$ 107.6(2) C(101a)-Si(1)-C(102a) 109.0(2)

Other Bond Angles

\begin{tabular}{ccc}
\hline & $\mathrm{n}=1$ & $\mathrm{n}=2$ \\
\hline $\mathrm{Si}(1)-\mathrm{C}(10 n)-\mathrm{C}(9 n)$ & $116.5(2)$ & $115.4(4)$ \\
$\mathrm{C}(9 n)-\mathrm{C}(8 n)-O(1 n)$ & $107.5(3)$ & $107.3(3)$ \\
$\mathrm{C}(7 n)-\mathrm{O}(1 n)-\mathrm{C}(8 n)$ & $116.2(3)$ & $116.0(3)$ \\
$\mathrm{O}(1 n)-\mathrm{C}(7 n)-O(2 n)$ & $122.4(4)$ & $123.3(5)$ \\
$\mathrm{C}(4 n)-\mathrm{C}(7 n)-O(1 n)$ & $112.8(3)$ & $112.2(4)$ \\
$\mathrm{C}(4 n)-\mathrm{C}(7 n)-O(2 n)$ & $124.8(4)$ & $124.5(5)$
\end{tabular}

a Deviations from the aryl mean plane $\left(\chi^{2}=5.4(1), 35.6(2)\right)$ : $C(1 n), 0.003(5), 0.017(4) ; C(2 n) 0.000(5),-0.009(4) ; C(3 n)-0.004(5)$, $-0.007(4) ; C(4 n)-0.001(5), 0.014(3) ; C(5 n) 0.003(5),-0.006(4)$; $C(6 n)-0.008(5),-0.010(4) ; C(7 n) 0.004(5), 0.076(4) ; I(1 n) 0.033(1)$, $0.160(1) \AA$.

Arl. However, as well as the desired product, a byproduct which is probably a carbonate-bridged "didendrimer" species, on the basis of its GPC(SEC) trace (which shows 
Table 3. Torsion Angles (deg) and Other Data Describing the Configuration at Silicon and the Conformation of $\left.\mathrm{G}_{0}-\mathrm{ArI}, \mathrm{Si}\left\{\left(\mathrm{CH}_{2}\right)_{3} \mathrm{O}_{2} \mathrm{CC}_{6} \mathrm{H}_{4} \mathrm{I}-4\right\}_{3}\right\}_{4}(4)^{\mathrm{a}}$

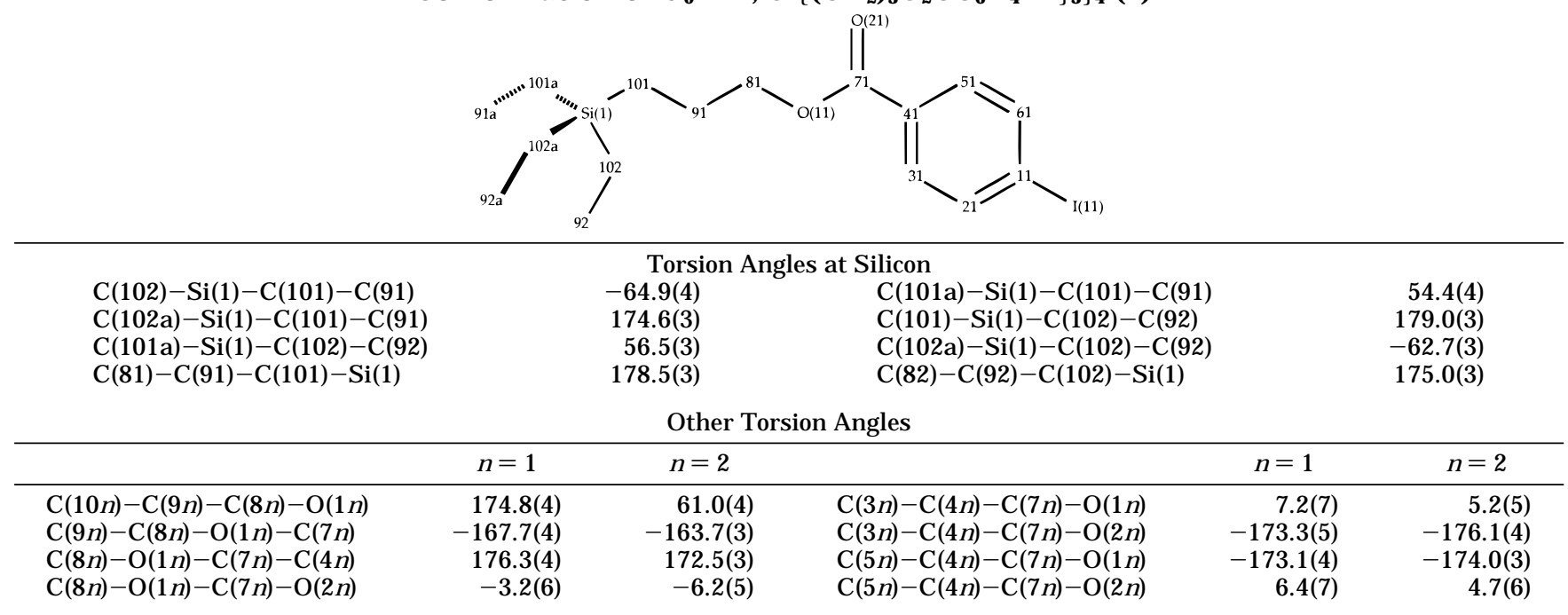

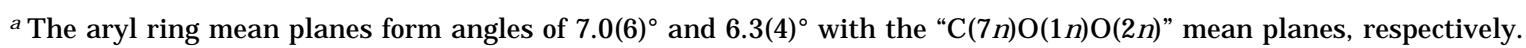

a rather broad molecular weight distribution with a tail into the higher molecular weight region), was also formed, as a result of incomplete consumption of carbonyldiimidazole (CDI) during the preparation of the acid imidazolide. Remaining CDI imidazolide leads inevitably to the formation of carbonate bridges between dendrimers bearing $\mathrm{OH}$ groups because in this respect it reacts as a phosgene equival ent. ${ }^{33}$ This prompted the use of 4-iodobenzoyl chloride as the activated acid derivative, and under the conditions used, a moderateyield, high-purity synthesis of $\mathrm{G}_{1}$-Arl was achieved (Scheme 2).

$\mathrm{G}_{0}$-Arl and $\mathrm{G}_{1}-\mathrm{Arl}$ were characterized by NMR $\left({ }^{1} \mathrm{H}\right.$, $\left.{ }^{13} \mathrm{C}\right)$ spectroscopy, exhibiting resonances with integration as expected: an AB pattern (aryl), $24\left(\mathrm{G}_{0}\right)$ or 72 $\left(\mathrm{G}_{1}\right)$ "outer" methylene protons as three well-resolved resonances. The GPC(SEC) trace of $\mathrm{G}_{1}$-Arl shows a narrow molecular weight distribution, with $M_{w} / M_{n}=$ 1.02. Differential scanning calorimetry (DSC) studies of $\mathrm{G}_{1}$-Arl show that it does not tend to crystallize with cooling, nor does it show any interesting phase behavior. However, its glass transition temperature, $T_{g}$, increased markedly to $17{ }^{\circ} \mathrm{C}$, compared with the polyol precursor $\left(\mathrm{T}_{\mathrm{g}}=-40^{\circ} \mathrm{C}\right) .{ }^{9} \mathrm{It}$ is the inner, flexible segments of the dendrimeric molecules which largely contribute to $T_{g},{ }^{34}$ and it is likely that the terminal iodoarene groups exert a substantially increased ordering effect on these inner segments compared to terminal hydroxyl groups. $\mathrm{G}_{0}-$ Arl and $\mathrm{G}_{1}$-Arl were characterized by el emental analysis, and $\mathrm{G}_{0}$-Arl was further characterized by X-ray cystallography (Figure 1, vide infra).

Reactions of $\mathbf{G}_{0}-\mathrm{Arl}$ and $\mathrm{G}_{1}$-Arl with $\mathrm{Pd}(\mathrm{dba})_{2}$ : Synthesis and Characterization of Complexes. The oxidative addition of substituted iodoarenes to $\mathrm{Pd}(0)$ precursors is well understood ${ }^{30}$ and is shown here to be applicable to the high-yield and high-purity synthesis of dendrimeric materials (Scheme 3 ).

It is possible that this reaction system may present difficulties with increasing generation number, due to

(33) Staab, H. A. Angew. Chem. 1962, 74, 407.

(34) (a) Wooley, K. L.; Hawker, C. J .; Pochan, j. M.; Fréchet, J . M. J . Macromol ecules 1993, 26, 1514. (b) Stutz, H.J . J . Polym. Sci. 1995, $33,333$. decreasing solubility of partially palladated high molecular mass dendrimer molecules. ${ }^{1 H}$ NMR spectra of the complexes show the expected $A B$ pattern for the phenyl ring protons, two signals for the $\mathrm{NMe}_{2}$ groups, and three well-resolved signals for "outer" methylene groups $\left(\mathrm{G}_{0}\right.$ and $\left.\mathrm{G}_{1}\right)$. The most characteristic feature of the ${ }^{13} \mathrm{C}$ NMR spectra is the lack of a signal at approximately 100 ppm, arising from $\mathrm{C}-\mathrm{I}$ in $\mathrm{G}_{0^{-}}$and $\mathrm{G}_{1^{-}}$ Arl, indicating, in conjunction with the "clean" $A B$ pattern in the ${ }^{1} \mathrm{H}$ NMR spectra, complete palladation. $\mathrm{G}_{1}$-ArPdl (tmeda) (Figure 2) displayed poor solubility in $\mathrm{CDCl}_{3}$, precipitating as an oil during spectroscopic measurements; thus the spectra of this complex were recorded in $\mathrm{CD}_{2} \mathrm{Cl}_{2}$. Elemental analyses of palladated $\mathrm{G}_{0}$ and $\mathrm{G}_{1}$ are in excellent agreement with calculated values, although for complexes of high molecular weight, very close agreement between calculated and observed values is expected even in the presence of minor amounts of impurities. Despite the crystalline nature of $\mathrm{G}_{0}-\mathrm{Arl}$, repeated attempts to grow crystals of $\mathrm{G}_{0}$ ArPdl (tmeda) suitable for X-ray crystallography have failed; the material generally preci pitates from solution as an oil.

Reactions of Model Palladium Complexes: Attempts at Transmetalation. Model complexes 2 and $\mathbf{3}$ were synthesized in order to attempt the devel opment of transmetalation procedures suitable for the palladated dendrimers. Methylation of Pdl Ar(tmeda) complexes with LiMe is generally straightforward, although it is not successful for aryl groups containing el ectronwithdrawing groups $\left(\mathrm{NO}_{2}\right.$, acetyl) at the 4-position. ${ }^{30 \mathrm{c}}$ In this study also, LiMe was an unsuitable reagent for the transmetalation of the model complexes, and studies of methylation have been extended to include $\mathrm{SnMe}_{4}$ and $\mathrm{Sn}(\mathrm{C} \sim \mathrm{N}) \mathrm{Me}_{3}[\mathrm{C} \sim \mathrm{N})=$ (8-(dimethylamino)-1-naphthyl]. ${ }^{31}$ The latter tetraorganotin complex contains an intramolecularly coordinated $\mathrm{C} \sim \mathrm{N}$ moiety, and in the triphenyl anal ogue, $\mathrm{X}$-ray crystallographic studies have shown that the axial $\mathrm{Sn}-\mathrm{C}$ bond trans to the nitrogen donor atom is significantly longer than the equatorial $\mathrm{Sn}-\mathrm{C}$ bonds. Thus, this reagent is expected to have potential as a methylating reagent, via transfer of the axial methyl group, and in preliminary studies, this 
complex has been shown to transmetallate dihalide complexes of palladium and platinum and to undergo very rapid redistribution reactions with triorganotin halides. ${ }^{31}$ The tmeda complex was reacted with all three reagents, although not at reflux temperatures, since diorgano(tmeda)pal ladium(II) complexes containing substituted aryl groups are thermally unstable. ${ }^{35}$ None of the transmetalation reactions were successful.

The complex of bpy (3) was reacted with $\mathrm{SnMe}_{4}$ and $\mathrm{Sn}(\mathrm{C} \sim \mathrm{N}) \mathrm{Me}_{3}$ at room temperature and reflux in tetrahydrofuran, but not at all with $\mathrm{LiMe}$, since previous attempts to transmetallate monoorganopalladium(II) halide complexes of bpy have not been successful. The inability to generate a diorganopalladium(II) complex using the model complexes precluded attempts at the functional ization of the ester-containing dendrimer, and model complexes, reported herein.

X-ray Crystallography. Molecules of compound $\mathbf{4}$ in the solid state have a crystallographic 2-fold axis through the silicon atom (Figure 1 ) with tetrahedral geometry at silicon $\left[107.6(2)-112.7(2)^{\circ}, \mathrm{Si}-\mathrm{C}=1.868(4)\right.$, $1.869(5) \AA]$ (Table 2). The two crystallographically independent arms " $\left(\mathrm{CH}_{2}\right)_{3} \mathrm{O}_{2} \mathrm{CC}_{6} \mathrm{H}_{4} \mathrm{I}-4$ " have similar conformations except near the silicon atom (Table 3 ), exhibiting corresponding torsion angles within $4^{\circ}$ except for $\mathrm{C}(10 n)-\mathrm{C}(9 \mathrm{n})-\mathrm{C}(8 \mathrm{n})-\mathrm{O}(1 \mathrm{n})\left[174.8(4)\right.$ and $\left.61.0(4)^{\circ}\right]$. The crystal structure shows no special packing features, and there are no solvent accessible voids. The shortest intermolecular $1 \cdots \mid$ interaction is 4.1440(6) $\AA$.

(35) Hoare, J . L.; Hovestad, N.; J astrzebski, J . T. B. H.; Canty, A. J .; Smeets, W. J .; Spek, A. L.; van Koten, G. Manuscript in preparation.

\section{Concluding Remarks}

The results reported here indicate that dendrimeric polyols are useful precursors for the synthesis of iodoarene-functionalized dendrimers. Oxidative addition reactions of $\mathrm{Pd}(\mathrm{dba})_{2} /$ tmeda with model reagents proceed in high yield, and this reaction chemistry also allows the synthesis of the fully palladated dendrimer 7 (Figure 2 ) in $81 \%$ yield. Dendrimer $\mathbf{7}$ is air stable at ambient temperature in $\mathrm{CH}_{2} \mathrm{Cl}_{2}$ solution and in the solid state. The crystal structure of $\mathrm{G}_{0}$-Arl (Figure 1) provides a model for the structure of the core and chain geometry for iodoarene-functionalized carbosilane dendrimers. The chain conformations in the sol id state for this molecule are probably dictated by packing require ments.

Acknowledgment. We thank the Australian Research Council, the Netherlands Foundation for Chemical Research (SON), the Netherlands Organisation for Scientific Research (NWO), the Fonds der Chemischen Industrie, and the Deutsche Forschungsgemeinschaft (Schwerpunktprogramm Siliciumchemie) for financial support. Financial support for visits by J.L.H. (University of Tasmania, Utrecht University), K.L. (Utrecht University), and A.J.C. (Netherlands Institute for Catalysis) is gratefully acknowledged.

Supporting Information Available: Listings of atom coordinates, thermal parameters, hydrogen atom parameters, and ligand geometry for the complexes (15 pages). Ordering information is given on any current masthead page.

OM $970343 E$ 